\title{
The Spectrum and Eigenvectors of the Laplacian Matrices of the Brualdi-Li Tournament Digraphs
}

\author{
Xiaogen Chen \\ School of Information Science and Technology, Zhanjiang Normal University, Zhanjiang, Guangdong 524048, China \\ Correspondence should be addressed to Xiaogen Chen; oamc@163.com
}

Received 26 February 2014; Revised 5 May 2014; Accepted 6 May 2014; Published 14 May 2014

Academic Editor: Chein-Shan Liu

Copyright (C) 2014 Xiaogen Chen. This is an open access article distributed under the Creative Commons Attribution License, which permits unrestricted use, distribution, and reproduction in any medium, provided the original work is properly cited.

Let $m \geq 1$ be an integer, let $\mathscr{B}_{2 m}$ denote the Brualdi-Li matrix of order $2 m$, and let $\mathscr{L} \mathscr{B}_{2 m}$ denote the Laplacian matrices of BrualdiLi tournament digraphs. We obtain the eigenvalues and eigenvectors of $\mathscr{L} \mathscr{B}_{2 m}$.

\section{Introduction}

The Laplacian spectral theory is currently not only a hot research direction of spectral graph theory but also one of the research directions of combined matrix theory. The Laplacian spectrum of a graph is of importance in graph theory, matrix theory, and the definite solution of partial differential equations. It also has applications in quantum chemistry, biology, and complex network. Therefore, it has important theoretical and practical values to study the Laplacian eigenvalues and eigenvectors of graphs. The Laplacian spectrum of graph has attracted the attention of researchers; see [1-5] and so on. We follow $[1,6]$ for terminology and notations.

Let $G$ be a digraph of order $n$ with vertex set $V(G)$ and arc set $E(G)$, where $V(G)=\left\{v_{1}, v_{2}, \ldots, v_{n}\right\}$. The adjacency matrix of $G$ is the $(0,1)$ matrix $A(G)=\left(a_{i j}\right)$ of order $n$, where $a_{i j}=1$ if there is an arc from $v_{i}$ to $v_{j}$ and $a_{i j}=0$ otherwise. The digraph $G$ is called the associated digraph of matrix $A(G)$. Let $D(G)=\operatorname{diag}\left(d_{1}, d_{2}, \ldots, d_{n}\right)$, the diagonal matrix with vertex outdegrees $d_{1}, d_{2}, \ldots, d_{n}$ of $v_{1}, v_{2}, \ldots, v_{n}$. $L(G)=D(G)-A(G)$ is called the Laplacian matrix of the digraph $G$. The characteristic polynomial of the adjacency matrix $A(G)$, that is, $P(G, \lambda)=P(A(G), \lambda)=\operatorname{det}(\lambda I-A(G))$, is called the characteristic polynomial of the digraph $G$. The equation $\operatorname{det}(\lambda I-A(G))=0$ has $n$ complex roots and these roots are called the eigenvalues of $A(G)$. Suppose the distinct eigenvalues of $L(G)$ are denoted by $\lambda_{1}, \lambda_{2}, \ldots, \lambda_{r} \in \mathbb{C}$ with corresponding algebraic multiplicities $\mu_{\lambda_{1}}, \mu_{\lambda_{2}}, \ldots, \mu_{\lambda_{r}}$, where $\mu_{\lambda_{i}}$ is a nonnegative integer $i=1,2, \ldots, r$.
$L S=\left(\begin{array}{cccc}\lambda_{1} & \lambda_{2} & \cdots & \lambda_{r} \\ \mu_{\lambda_{1}} & \mu_{\lambda_{2}} & \cdots & \mu_{\lambda_{r}}\end{array}\right)$ is called the Laplacian spectrum of digraph $G$. The Laplacian spectral radius of $G$ is the largest modulus of an eigenvalue of $L(G)$, denoted by $\rho(L(G))$. The symbol $\mathbb{C}$ will denote the complex field. Let $\lambda \in \mathbb{C}$ be an eigenvalue of matrix $A$. There is vector $x \neq 0$ satisfying $A x=\lambda x$, and then $x$ is called the eigenvectors of matrix $A$ corresponding to $\lambda$.

A tournament matrix of order $n$ is a $(0,1)$ matrix $T_{n}$ satisfying the equation $T_{n}+T_{n}^{t}=J_{n}-I_{n}$, where $J_{n}$ is the all ones matrix, $I_{n}$ is the identity matrix, and $T_{n}^{t}$ is the transpose of $T_{n}$. Let

$$
\mathscr{B}_{2 m}=\left(\begin{array}{cc}
U_{m} & U_{m}^{t} \\
I_{m}+U_{m}^{t} & U_{m}
\end{array}\right),
$$

where $U_{m}$ is strictly upper triangular tournament matrix (all of whose entries above the main diagonal are equal to one); that is,

$$
U_{m}=\left(\begin{array}{ccccc}
0 & 1 & 1 & \cdots & 1 \\
0 & 0 & 1 & \cdots & 1 \\
\vdots & \ddots & \ddots & \ddots & \vdots \\
0 & 0 & \cdots & 0 & 1 \\
0 & 0 & \cdots & 0 & 0
\end{array}\right)_{m \times m}
$$

is the tournament matrix of order $2 m$.

The matrix $\mathscr{B}_{2 m}$ has been dubbed by the Brualdi-Li matrix. The associated digraph of matrix $\mathscr{B}_{2 m}$ is called the Brualdi-Li tournament digraph. In 1983 Brualdi and Li 
conjectured that the maximal spectral radius for tournaments of order $2 m$ is attained by the Brualdi-Li matrix [7]. This conjecture has been confirmed in [8]. The properties of the Brualdi-Li matrix have been investigated in [9-14].

In this paper, we obtain the spectrum and eigenvectors of the Laplacian matrices of the Brualdi-Li tournament digraphs.

Theorem 1. Let $m \geq 1$ be an integer, and let $\delta \mathscr{L} \mathscr{B}_{2 m}$ be the Laplacian spectrum of the Brualdi-Li tournament digraph. Then



where $\lfloor a\rfloor$ is the floor of number $a, \lambda_{k}=m-i \cot ((\pi+$ $2 k \pi) / 2 m$ ), and $\overline{\lambda_{k}}$ is the conjugate complex number of $\lambda_{k}$, $k=0,1,2, \ldots,\lfloor m / 2\rfloor-1$.

Theorem 2. Let $m \geq 1$ be an integer, and let $\xi=\left(\begin{array}{c}v \\ w\end{array}\right) \neq 0$ be the eigenvector of $\mathscr{L} \mathscr{B}_{2 m}$ corresponding to $\lambda$, where $v=$ $\left(v_{1}, v_{2}, \ldots, v_{m}\right)^{t}, w=\left(w_{1}, w_{2}, \ldots, w_{m}\right)^{t}$; then

(1) if $\lambda=m$, then

$\xi_{m}=\left(\begin{array}{l}0 \\ 1\end{array}\right)$ is the eigenvector of $\mathscr{L} \mathscr{B}_{2}$ corresponding to $\lambda=m=1$,

$\xi_{m}=(1,-1,1,-1)^{t}$ is the eigenvector of $\mathscr{L} \mathscr{B}_{4}$ corresponding to $\lambda=m=2$,

$\xi_{m}=\sum_{k=2}^{m} l_{k} \xi_{m_{k}}$ is the eigenvector of $\mathscr{L} \mathscr{B}_{2 m}$ corresponding to $\lambda=m>2$,

where $l_{k}$ is an arbitrary constant, $k=2,3, \ldots, m$,

$$
\left(\xi_{m_{2}}, \xi_{m_{3}}, \ldots, \xi_{m_{m}}\right)=\left(\begin{array}{cc}
\mathbf{1}_{m-2}^{t} & 1 \\
-I_{m-2} & 0 \\
0 & -1 \\
\mathbf{1}_{m-2}^{t} & 1 \\
-I_{m-2} & 0 \\
0 & -1
\end{array}\right)_{2 m \times(m-1)}
$$

(2) if $\lambda \neq m$, then

$$
\begin{array}{r}
v_{k}=\frac{1}{2(m-\lambda)}\left(1-\lambda\left(\frac{m-1-\lambda}{m+1-\lambda}\right)^{k-1}\right), \\
w_{k}=\frac{1}{2(m-\lambda)}\left(1+\lambda\left(\frac{m-1-\lambda}{m+1-\lambda}\right)^{k}\right), \\
k=1,2, \cdots, m .
\end{array}
$$

Corollary 3. Let $m \geq 1$ be an integer, and let $\rho\left(\mathscr{L} \mathscr{B}_{2 m}\right)$ be the Laplacian spectral radius of the Brualdi-Li tournament digraph. Then

$$
\rho\left(\mathscr{L} \mathscr{B}_{2 m}\right)=\sqrt{m^{2}+\cot ^{2} \frac{\pi}{2 m}} .
$$

Proof. By Theorem 1,

$$
\begin{aligned}
\rho\left(\mathscr{L} \mathscr{B}_{2 m}\right) & =\max _{0 \leq k \leq\lfloor m / 2\rfloor-1}\left\{0, m,\left|m \pm i \cot \frac{\pi+2 k \pi}{2 m}\right|\right\} \\
& =\sqrt{m^{2}+\cot ^{2} \frac{\pi}{2 m}} .
\end{aligned}
$$

Corollary 4. Let $m \geq 1$ be an integer, then

(1) if $m>1$ is odd, then $\mathscr{L}_{2}$ is not diagonalizable,

(2) if $m$ is even or $m=1$, then $\mathscr{L}_{2 m}$ is diagonalizable.

\section{Some Lemmas}

Fundamental Theorem of Algebra. Every nonzero, singlevariable, degree $n$ polynomial with complex coefficients has, counted with multiplicity, exactly $n$ roots.

Complex Conjugate Root Theorem. If $P$ is a polynomial in one variable with real coefficients and $a+b i$ is a root of $P$ with $a$ and $b$ real numbers, then its complex conjugate $a-b i$ is also a root of $P$, where $i^{2}=-1$.

The symbol $\mathscr{L} \mathscr{B}_{2 m}$ denotes the Laplacian matrix of the Brualdi-Li tournament digraph. Clearly, $\mathscr{L} \mathscr{B}_{2 m}=$ $\left(\begin{array}{cc}(m-1) I_{m}-U_{m} & -U_{m}^{t} \\ -I_{m}-U_{m}^{t} & m I_{m}-U_{m}\end{array}\right)$.

Lemma 5. Let $m>1$ be an integer, and $X=\left(1, x, x^{2}\right.$, $\left.\ldots, x^{m-1}\right)^{t}$, where $x \neq 1$ is real variable. Then

$$
\begin{aligned}
& \text { (1) } X^{t} U_{m}=-\frac{1}{1-x} X^{t}+\frac{1}{1-x} \mathbf{1}_{m}^{t}, \\
& \text { (2) } X^{t} U_{m}^{t}=\frac{x}{1-x} X^{t}-\frac{x^{m}}{1-x} \mathbf{1}_{m}^{t} .
\end{aligned}
$$

Proof. Consider

(1) $X^{t} U_{m}$

$$
\begin{aligned}
& =\left(1, x, x^{2}, \ldots, x^{m-1}\right) U_{m} \\
& =\left(0, \sum_{k=0}^{0} x^{k}, \sum_{k=0}^{1} x^{k}, \sum_{k=0}^{2} x^{k},\right. \\
& \\
& \left.\quad \sum_{k=0}^{3} x^{k}, \ldots, \sum_{k=0}^{m-3} x^{k}, \sum_{k=0}^{m-2} x^{k}\right) \\
& =\left(\frac{1-1}{1-x}, \frac{1-x}{1-x}, \frac{1-x^{2}}{1-x}, \frac{1-x^{3}}{1-x}, \ldots,\right. \\
& \left.\frac{1-x^{m-2}}{1-x}, \frac{1-x^{m-1}}{1-x}\right) \\
& =-\frac{1}{1-x} X^{t}+\frac{1}{1-x} \mathbf{1}_{m}^{t} .
\end{aligned}
$$


(2) $X^{t} U_{m}^{t}$

$$
\begin{aligned}
& =\left(1, x, x^{2}, \ldots, x^{m-1}\right) U_{m}^{t} \\
& =\left(\sum_{k=1}^{m-1} x^{k}, \sum_{k=2}^{m-1} x^{k}, \ldots, \sum_{k=m-2}^{m-1} x^{k}, \sum_{k=m-1}^{m-1} x^{k}, 0\right) \\
& =\left(\frac{x-x^{m}}{1-x}, \frac{x^{2}-x^{m}}{1-x}, \ldots, \frac{x^{m-2}-x^{m}}{1-x},\right. \\
& \left.\frac{x^{m-1}-x^{m}}{1-x}, \frac{x^{m}-x^{m}}{1-x}\right) \\
& =\frac{x}{1-x} X^{t}-\frac{x^{m}}{1-x} \mathbf{1}_{m}^{t} .
\end{aligned}
$$

Lemma 6. Let $m>1$ be an integer, $\lambda \neq m$, let $\lambda \in \mathbb{C}$ be an arbitrary eigenvalue of $\mathscr{L}_{2 m}$, and let $\xi=\left(\begin{array}{c}v \\ w\end{array}\right) \neq 0$ be the eigenvector of $\mathscr{L} \mathscr{B}_{2 m}$ corresponding to $\lambda$, where $v=\left(v_{1}, v_{2}, \ldots, v_{m}\right)^{t}$, $w=\left(w_{1}, w_{2}, \ldots, w_{m}\right)^{t}$. Let $X=\left(1, x, x^{2}, \ldots, x^{m-1}\right)^{t}, f(x)=$ $\sum_{k=1}^{m} v_{k} x^{k-1}$, and $g(x)=\sum_{k=1}^{m} w_{k} x^{k-1}$, where $x$ is real variable. Then

(1) $f(x)$

$$
\begin{aligned}
= & \left((m+1-\lambda) a+(a+b)\left(x+x^{2}+\cdots+x^{m-1}\right)\right. \\
& \left.+(a-b(m-\lambda)) x^{m}\right) \\
& \times((m-\lambda)((m+1-\lambda)-(m-1-\lambda) x))^{-1},
\end{aligned}
$$

(2) $g(x)$

$$
\begin{aligned}
= & \left(a+b(m-\lambda)+(a+b)\left(x+x^{2}+\cdots+x^{m-1}\right)\right. \\
& \left.-a(m-1-\lambda) x^{m}\right) \\
& \times((m-\lambda)((m+1-\lambda)-(m-1-\lambda) x))^{-1},
\end{aligned}
$$

where $a=\mathbf{1}_{m}^{t} v, b=\mathbf{1}_{m}^{t} w$.

Proof. If $\lambda(\neq m)$ is an eigenvalue, with eigenvector $\xi=\left(\begin{array}{c}v \\ w\end{array}\right)$, of $\mathscr{L} \mathscr{B}_{2 m}$, then $\mathscr{L} \mathscr{B}_{2 m} \xi=\lambda \xi$ expands to

$$
\left(\begin{array}{cc}
(m-1) I_{m}-U_{m} & -U_{m}^{t} \\
-I_{m}-U_{m}^{t} & m I_{m}-U_{m}
\end{array}\right)\left(\begin{array}{c}
v \\
w
\end{array}\right)=\lambda\left(\begin{array}{c}
v \\
w
\end{array}\right) .
$$

Therefore,

$$
\begin{gathered}
\left((m-1) I_{m}-U_{m}\right) v-U_{m}^{t} w=\lambda v \\
\left(-I_{m}-U_{m}^{t}\right) v+\left(m I_{m}-U_{m}\right) w=\lambda w .
\end{gathered}
$$

We have

$$
\begin{gathered}
X^{t}\left((m-1) I_{m}-U_{m}\right) v-X^{t} U_{m}^{t} w=\lambda X^{t} v \\
X^{t}\left(-I_{m}-U_{m}^{t}\right) v+X^{t}\left(m I_{m}-U_{m}\right) w=\lambda X^{t} w .
\end{gathered}
$$

According to Lemma 5, we have

$$
\begin{gathered}
((m-1-\lambda)(1-x)+1) f(x)-x g(x)=a-b x^{m} \\
-f(x)+((m-\lambda)(1-x)+1) g(x)=b-a x^{m}
\end{gathered}
$$

Notice that this equation holds for $x=1$ too. Consider

$$
\begin{aligned}
& D=\operatorname{det}\left(\begin{array}{cc}
(m-1-\lambda)(1-x)+1 & -x \\
-1 & (m-\lambda)(1-x)+1
\end{array}\right) \\
& =((m-1-\lambda)(1-x)+1)((m-\lambda)(1-x)+1)-x \\
& =(m-\lambda)(1-x)((m+1-\lambda)-(m-1-\lambda) x) \text {, } \\
& D_{f}=\operatorname{det}\left(\begin{array}{cc}
a-b x^{m} & -x \\
b-a x^{m} & (m-\lambda)(1-x)+1
\end{array}\right) \\
& =\left(a-b x^{m}\right)((m-\lambda)(1-x)+1)+x\left(b-a x^{m}\right) \\
& =a(m+1-\lambda)+(b-a(m-\lambda)) x \\
& -b(m+1-\lambda) x^{m}+(b(m-\lambda)-a) x^{m+1} \\
& =(1-x)\left(a(m+1-\lambda)+(a+b)\left(x+\cdots+x^{m-1}\right)\right. \\
& \left.+(a-b(m-\lambda)) x^{m}\right) \\
& D_{g}=\operatorname{det}\left(\begin{array}{cc}
(m-1-\lambda)(1-x)+1 & a-b x^{m} \\
-1 & b-a x^{m}
\end{array}\right) \\
& =\left(b-a x^{m}\right)((m-1-\lambda)(1-x)+1)+\left(a-b x^{m}\right) \\
& =a+b(m-\lambda)-b(m-1-\lambda) x \\
& -(b+a(m-\lambda)) x^{m}+a(m-1-\lambda) x^{m+1} \\
& =(1-x)\left(a+b(m-\lambda)+(a+b)\left(x+\cdots+x^{m-1}\right)\right. \\
& \left.-a(m-1-\lambda) x^{m}\right) \text {. }
\end{aligned}
$$

By Cramer's rule,

(1) $f(x)$

$$
\begin{aligned}
= & \left((m+1-\lambda) a+(a+b)\left(x+x^{2}+\cdots+x^{m-1}\right)\right. \\
& \left.+(a-b(m-\lambda)) x^{m}\right) \\
& \times((m-\lambda)((m+1-\lambda)-(m-1-\lambda) x))^{-1},
\end{aligned}
$$

(2) $g(x)$

$$
\begin{aligned}
= & \left(a+b(m-\lambda)+(a+b)\left(x+x^{2}+\cdots+x^{m-1}\right)\right. \\
& \left.-a(m-1-\lambda) x^{m}\right) \\
& \times((m-\lambda)((m+1-\lambda)-(m-1-\lambda) x))^{-1} .
\end{aligned}
$$

We are done. 
Lemma 7. Under the assumptions and in the notation of Lemma 6 ,

$$
a+b=\mathbf{1}_{m}^{t} v+\mathbf{1}_{m}^{t} w \neq 0, \quad a=\mathbf{1}_{m}^{t} v \neq 0, \quad b=\mathbf{1}_{m}^{t} w \neq 0 .
$$

Proof. In Lemma 6(1), by setting $x=1$, we have

$$
\begin{aligned}
a & =f(1) \\
& =\frac{(m+1-\lambda) a+(a+b)(m-1)+(a-b(m-\lambda))}{(m-\lambda)((m+1-\lambda)-(m-1-\lambda))} .
\end{aligned}
$$

Since $\lambda \neq m$, it follows that

$$
(a+b)(1-\lambda)=2 a .
$$

As we all know that the eigenvalues of a real skew-symmetric matrix are all pure imaginary or nonzero, hence $\operatorname{det}((1-$ $\left.m) I_{m}+U-U^{t}\right) \neq 0$, where $m>1$. Since

$$
\begin{aligned}
\operatorname{det} & \left(1 I_{m}-\mathscr{L} \mathscr{B}_{2 m}\right) \\
= & \operatorname{det}\left(\begin{array}{cc}
(2-m) I_{m}+U_{m} & U_{m}^{t} \\
I_{m}+U_{m}^{t} & (1-m) I_{m}+U_{m}
\end{array}\right) \\
= & \operatorname{det}\left(\begin{array}{cc}
(2-m) I_{m}+U_{m} & (2-m) I_{m}+U_{m}+U_{m}^{t} \\
I_{m}+U_{m}^{t} & (2-m) I_{m}+U_{m}+U_{m}^{t}
\end{array}\right) \\
= & \operatorname{det}\left(\begin{array}{cc}
(1-m) I_{m}+U-U^{t} & 0 \\
I_{m}+U_{m}^{t} & (1-m) I_{m}+J m
\end{array}\right) \\
= & \operatorname{det}\left((1-m) I_{m}+U-U^{t}\right) \operatorname{det}\left((1-m) I_{m}+J m\right) \\
= & (1-m)^{m-1} \operatorname{det}\left((1-m) I_{m}+U-U^{t}\right) \neq 0,
\end{aligned}
$$

hence $\lambda \neq 1$. Notice that $(a+b)(1-\lambda)=2 a$ and if $a+b=0$, then $a=0$ and $b=(a+b)-a=0$. By Lemma 6 ,

$$
\begin{aligned}
& f(x)=\sum_{k=1}^{m} v_{k} x^{k-1} \equiv 0, \\
& g(x)=\sum_{k=1}^{m} w_{k} x^{k-1} \equiv 0,
\end{aligned}
$$

for arbitrary real variable $x$. It is not possible. Hence $a+b \neq 0$. It is easy to see that $a \neq 0$ and $b \neq 0$.

Lemma 8 (see [15]). If $A$ is a square matrix, then,

(1) for every eigenvalue of $A$, the geometric multiplicity is less than or equal to the algebraic multiplicity,

(2) $A$ is diagonalizable if and only if the geometric multiplicity of every eigenvalue is equal to the algebraic multiplicity.

\section{Proof of Theorem 1}

Let $\lambda \in \mathbb{C}$ be an arbitrary eigenvalue of $\mathscr{L}_{2}$, and let $\xi=\left(\begin{array}{c}v \\ w\end{array}\right) \neq 0$ be the eigenvector of $\mathscr{L} \mathscr{B}_{2 m}$ corresponding to $\lambda$, where $v=\left(v_{1}, v_{2}, \ldots, v_{m}\right)^{t}, w=\left(w_{1}, w_{2}, \ldots, w_{m}\right)^{t}$.
For $m=1$, then $\mathscr{B}_{2}=\left(\begin{array}{ll}0 & 0 \\ 1 & 0\end{array}\right), \mathscr{L} \mathscr{B}_{2}=\left(\begin{array}{cc}0 & 0 \\ -1 & 1\end{array}\right)$. Obviously, $\lambda_{1}=0, \lambda_{2}=1$ are eigenvalues of $\mathscr{L} \mathscr{B}_{2}$ and $\mu_{0}=\mu_{1}=1$. Theorem 1 holds.

For $m>1$, using the previous assumptions and notation, obviously, $P\left(\mathscr{L} \mathscr{B}_{2 m}, 0\right)=\operatorname{det}\left(0 I_{2 m}-\mathscr{L} \mathscr{B}_{2 m}\right)=0$, by definition, $\lambda=0$ is an eigenvalue of $\mathscr{L} \mathscr{B}_{2 m}, \mu_{0} \geq 1$.

Note that $P\left(\mathscr{L} \mathscr{B}_{2 m}, m\right)=\operatorname{det}\left(m I_{2 m}-\mathscr{L} \mathscr{B}_{2 m}\right)=0$, and by definition, $\lambda=m$ is an eigenvalue of $\mathscr{L} \mathscr{B}_{2 m}$. By simple calculation, the rank of matrix $m I_{2 m}-\mathscr{L} \mathscr{B}_{2 m}$ is equal to $m+1$. By Lemma $8(1) \mu_{m} \geq 2 m-(m+1)=m-1$.

For $\lambda \neq m$, by Lemma 7, we set $a=\mathbf{1}_{m}^{t} v, a+b=\mathbf{1}_{m}^{t} v+$ $\mathbf{1}_{m}^{t} w=1$; hence $b=1-\mathbf{1}_{m}^{t} v=1-a$. In Lemma 6(1), by setting $x=1$, we have

$$
\begin{aligned}
a & =\mathbf{1}_{m}^{t} v=f(1) \\
& =\frac{(m+1-\lambda) a+m-1+a+(a-1)(m-\lambda)}{(m-\lambda)((m+1-\lambda)-(m-1-\lambda))} \\
& =\frac{(m-\lambda)(2 a-1)+m+2 a-1}{2(m-\lambda)} .
\end{aligned}
$$

Hence

$$
a=\frac{1-\lambda}{2} \text {. }
$$

Denoting $f_{0}=(m+1-\lambda) a, f_{1}=f_{2}=\cdots=f_{m-1}=1, f_{m}=$ $a+(a-1)(m-\lambda), c=(m-\lambda)(m+1-\lambda)$, and $d=(m-1-$ $\lambda) /(m+1-\lambda)$, we have

$f(x)$

$$
\begin{aligned}
= & \sum_{k=1}^{m} v_{k} x^{k-1} \\
= & \left((m+1-\lambda) a+x+x^{2}+\cdots+x^{m-1}\right. \\
& \left.+(a+(a-1)(m-\lambda)) x^{m}\right) \\
& \times((m-\lambda)((m+1-\lambda)-(m-1-\lambda) x))^{-1} \\
= & \frac{1}{c} \frac{1}{(1-d x)} \sum_{k=0}^{m} f_{k} x^{k}=\frac{1}{c} \sum_{k=0}^{\infty} d^{k} x^{k} \sum_{k=0}^{m} f_{k} x^{k} \\
= & \frac{1}{c} \sum_{k=0}^{\infty}\left(\sum_{j=0}^{k} f_{j} d^{k-j}\right) x^{k} .
\end{aligned}
$$

It must be that

$$
v_{k}=\frac{1}{c} \sum_{j=0}^{k-1} f_{j} d^{k-1-j}, \quad k=1,2, \ldots, m .
$$

Therefore

$$
\begin{aligned}
a & =\sum_{k=1}^{m} v_{k}=\frac{1}{c} \sum_{k=1}^{m} \sum_{j=0}^{k-1} f_{j} d^{k-1-j} \\
& =\frac{1}{c} \sum_{k=1}^{m}\left(f_{0} d^{k-1}+\sum_{j=1}^{k-1} d^{k-1-j}\right)
\end{aligned}
$$




$$
\begin{aligned}
= & \frac{1}{c} \sum_{k=1}^{m}\left(f_{0} d^{k-1}+\frac{1-d^{k-1}}{1-d}\right) \\
= & \frac{1}{c(1-d)^{2}} \\
& \times\left(f_{0}\left(1-d^{m}\right)(1-d)+m(1-d)-1+d^{m}\right) \\
= & \frac{1}{c(1-d)^{2}} \\
& \times\left(\left(1-f_{0}(1-d)\right) d^{m}+\left(f_{0}+m\right)(1-d)-1\right) .
\end{aligned}
$$

That is, $a c(1-d)^{2}=\left(1-f_{0}(1-d)\right) d^{m}+\left(f_{0}+m\right)(1-d)-1$.

Since $a=(1-\lambda) / 2, f_{0}=(m+1-\lambda) a, c=(m-\lambda)(m+1-\lambda)$, and $d=(m-1-\lambda) /(m+1-\lambda)$, then

$$
\begin{gathered}
\left(1-f_{0}(1-d)\right) d^{m}=a c(1-d)^{2}-\left(f_{0}+m\right)(1-d)+1, \\
\left(1-\frac{2 a(m+1-\lambda)}{m+1-\lambda}\right) d^{m} \\
=\frac{4 a(m-\lambda)}{m+1-\lambda}-\frac{2((m+1-\lambda) a+m)}{m+1-\lambda}+1, \\
\lambda d^{m}=-\lambda .
\end{gathered}
$$

Denote $\theta_{k}=(\pi+2 k \pi) / 2 m, k=0,1,2, m-1$. As $\lambda \neq 0, \lambda-$ $m \neq 0$, we have $d=e^{2 i \theta_{k}}$; that is, $(m-1-\lambda) /(m+1-\lambda)=e^{2 i \theta_{k}}$, where $i^{2}=-1$.

Therefore,

$$
\lambda=\lambda_{k}=m-\frac{1+e^{2 i \theta_{k}}}{1-e^{2 i \theta_{k}}}=m-\frac{i \sin 2 \theta_{k}}{1-\cos 2 \theta_{k}}=m-i \cot \theta_{k},
$$

where $1-e^{2 i \theta_{k}} \neq 0, k=0,1,2, \ldots, m-1$.

Note that if $m$ is odd, by $d^{m}=((m-1-\lambda) /(m+1-\lambda))^{m}=$ -1 , then $(m-1-\lambda) /(m+1-\lambda)=-1$; hence $\lambda=m$. It is an eigenvalue of $\mathscr{L} \mathscr{B}_{2 m}$.

Furthermore, $\mu_{\lambda_{k}} \geq 1$. Note that $\overline{\lambda_{k}}=\lambda_{m-1-k}, k=$ $0,1,2, \ldots,\lfloor m / 2\rfloor-1$.

To sum up, by fundamental theorem of algebra and complex conjugate root theorem, for $m \geq 1$, we obtain the following conclusions.

If $m$ is odd, then $\mu_{0}=\mu_{\lambda_{k}}=\mu_{\overline{\lambda_{k}}}=1$ and $\mu_{m}=m=$ $\lfloor(m-1) / 2\rfloor+\lfloor(m+1) / 2\rfloor$.

If $m$ is even, then $\mu_{0}=\mu_{\lambda_{k}}=\mu_{\overline{\lambda_{k}}}=1$ and $\mu_{m}=m-1=$ $\lfloor(m-1) / 2\rfloor+\lfloor(m+1) / 2\rfloor$.

Consider $k=0,1,2, \ldots,\lfloor m / 2\rfloor-1$. We complete the proof of Theorem 1 .

\section{Proofs of Theorem 2 and Corollary 4}

It is easy to see that the distinct eigenvalues of $\mathscr{L} \mathscr{B}_{2 m}$ are $0, \lambda_{k}=m-i \cot ((\pi+2 k \pi) / 2 m), \overline{\lambda_{k}}, m$, with corresponding algebraic multiplicities $1,1,1,\lfloor(m-1) / 2\rfloor+\lfloor(m+1) / 2\rfloor, k=$ $1,2, \ldots,\lfloor m / 2\rfloor-1$.

For $\lambda=m=1, \mathscr{L} \mathscr{B}_{2}=\left(\begin{array}{cc}0 & 0 \\ -1 & 1\end{array}\right)$ and, obviously, $\xi_{m}=\left(\begin{array}{l}0 \\ 1\end{array}\right)$ is the eigenvector of $\mathscr{L} \mathscr{B}_{2}$ corresponding to $\lambda=m=1$.

For $\lambda=m=2$,

$$
\mathscr{L} \mathscr{B}_{4}=\left(\begin{array}{cccc}
1 & -1 & 0 & 0 \\
0 & 1 & -1 & 0 \\
-1 & 0 & 2 & -1 \\
-1 & -1 & 0 & 2
\end{array}\right) .
$$

By simple calculation, $\xi_{m}=(1,-1,1,-1)^{t}$ is the eigenvector of $\mathscr{L} \mathscr{B}_{4}$ corresponding to $\lambda=m=2$.

For $\lambda=m>2$, let $\xi_{m}=\left(\begin{array}{c}v \\ w\end{array}\right) \neq 0$ be the eigenvector of $\mathscr{L} \mathscr{B}_{2 m}$ corresponding to $m$, and then $\mathscr{L} \mathscr{B}_{2 m} \xi_{m}=m \xi_{m}$ expands to

$$
\left(\begin{array}{cc}
I_{m}+U_{m} & U_{m}^{t} \\
I_{m}+U_{m}^{t} & U_{m}
\end{array}\right)\left(\begin{array}{c}
v \\
w
\end{array}\right)=0
$$

Equation (30) is equivalent to the following equation:

$$
\left(\begin{array}{cc}
I_{m} & \left(I+U_{m}\right)^{-1} U_{m}^{t} \\
0_{m} & U_{m}-\left(I_{m}-U_{m}^{t}\right)\left(I+U_{m}\right)^{-1} U_{m}^{t}
\end{array}\right)\left(\begin{array}{c}
v \\
w
\end{array}\right)=0 .
$$

By simple calculation,

$$
\begin{gathered}
\left(I+U_{m}\right)^{-1} U_{m}^{t}=\left(\begin{array}{cc}
-I_{m-1} & 0 \\
\mathbf{1}_{m-1}^{t} & 0
\end{array}\right), \\
U_{m}-\left(I_{m}-U_{m}^{t}\right)\left(I+U_{m}\right)^{-1} U_{m}^{t}=\left(\begin{array}{cc}
J_{m-1} & \mathbf{1}_{m-1} \\
0 & 0
\end{array}\right) .
\end{gathered}
$$

We obtain the solution of (30) as follows:

$$
\xi_{m}=\sum_{k=2}^{m} l_{k} \xi_{m_{k}}
$$

where $l_{k}$ is an arbitrary constant, $k=2,3, \ldots, m$, and

$$
\left(\xi_{m_{2}}, \xi_{m_{3}}, \ldots, \xi_{m_{m}}\right)=\left(\begin{array}{cc}
\mathbf{1}_{m-2}^{t} & 1 \\
-I_{m-2} & 0 \\
0 & -1 \\
\mathbf{1}_{m-2}^{t} & 1 \\
-I_{m-2} & 0 \\
0 & -1
\end{array}\right)_{2 m \times(m-1)}
$$

For $\lambda \neq m$, let $\xi=\left(\begin{array}{c}v \\ w\end{array}\right) \neq 0$ be the eigenvector of $\mathscr{L} \mathscr{B}_{2 m}$ corresponding to $\lambda(\neq m)$, where $v=\left(v_{1}, v_{2}, \ldots, v_{m}\right)^{t}, w=$ $\left(w_{1}, w_{2}, \ldots, w_{m}\right)^{t}$; then $\mathscr{L} \mathscr{B}_{2 m} \xi=\lambda \xi$ expands to

$$
\left(\begin{array}{cc}
(m-1) I_{m}-U_{m} & -U_{m}^{t} \\
-I_{m}-U_{m}^{t} & m I_{m}-U_{m}
\end{array}\right)\left(\begin{array}{c}
v \\
w
\end{array}\right)=\lambda\left(\begin{array}{c}
v \\
w
\end{array}\right)
$$

By Lemma 7, $\mathbf{1}_{m}^{t} v+\mathbf{1}_{m}^{t} w \neq 1$; we put $\mathbf{1}_{m}^{t} v+\mathbf{1}_{m}^{t} w=1$. By Theorem $1, a=\mathbf{1}_{m}^{t} v=(1-\lambda) / 2, b=\mathbf{1}_{m}^{t} w=1-a$. 
Denote $g_{0}=a+(1-a)(m-\lambda), g_{1}=g_{2}=\cdots=g_{m-1}=1$, and $g_{m}=-a(m-1-\lambda)$. By Lemma 6(2) we have

$$
\begin{aligned}
g(x) & \\
= & \sum_{k=1}^{m} w_{k} x^{k-1} \\
= & \left(a+(1-a)(m-\lambda)+x+x^{2}+\cdots+x^{m-1}\right. \\
& \times((m-\lambda)((m+1-\lambda)-(m-1-\lambda) x))^{-1} \\
= & \frac{1}{c} \frac{1}{(1-d x)} \sum_{k=0}^{m} g_{k} x^{k} \\
= & \frac{1}{c} \sum_{k=0}^{\infty} d^{k} x^{k} \sum_{k=0}^{m} g_{k} x^{k}=\frac{1}{c} \sum_{k=0}^{\infty}\left(\sum_{j=0}^{k} g_{j} d^{k-j}\right) x^{k} .
\end{aligned}
$$

It must be that

$$
w_{k}=\frac{1}{c} \sum_{j=0}^{k-1} g_{j} d^{k-1-j}, \quad k=1,2, \ldots, m .
$$

Hence

$$
\begin{aligned}
w_{k} & =\frac{1}{c} \sum_{j=0}^{k-1} g_{j} d^{k-1-j} \\
& =\frac{1}{c}\left(\frac{1-\lambda+(1+\lambda)(m-\lambda)}{2} d^{k-1}+\sum_{j=0}^{k-2} d^{j}\right) \\
& =\frac{1}{c}\left(\frac{1-\lambda+(1+\lambda)(m-\lambda)}{2} d^{k-1}+\frac{1-d^{k-1}}{1-d}\right) \\
& =\frac{1}{2(m-\lambda)}\left(1+\lambda\left(\frac{m-1-\lambda}{m+1-\lambda}\right)^{k}\right), \quad k=1,2, \ldots, m .
\end{aligned}
$$

In the proof of Theorem 1, we have

$$
\begin{aligned}
v_{k} & =\frac{1}{c} \sum_{j=0}^{k-1} f_{j} d^{k-1-j} \\
& =\frac{1}{c}\left(\frac{(m+1-\lambda)(1-\lambda)}{2} d^{k-1}+\sum_{j=0}^{k-2} d^{j}\right) \\
& =\frac{1}{c}\left(\frac{(m+1-\lambda)(1-\lambda)}{2} d^{k-1}+\frac{1-d^{k-1}}{1-d}\right) \\
& =\frac{1}{2(m-\lambda)}\left(1-\lambda\left(\frac{m-1-\lambda}{m+1-\lambda}\right)^{k-1}\right), \quad k=1,2, \ldots, m .
\end{aligned}
$$

We complete the proof of Theorem 2.
Let $\lambda$ be an eigenvalue of $\mathscr{L} \mathscr{B}_{2 m}$, and $\mu_{\lambda}$ and $\nu_{\lambda}$ are the algebraic multiplicity and the geometric multiplicity corresponding to $\lambda$, respectively.

If $m=1$, by Theorem 1 and Theorem 2 , and $\mu_{0}=\nu_{0}=$ $\mu_{1}=\nu_{1}=1$, then $\mathscr{L} \mathscr{B}_{2}$ is diagonalizable.

If $m>1$, by Theorem $1, \mu_{0}=1, \mu_{m}=\lfloor(m-1) / 2\rfloor+\lfloor(m+$ $1) / 2\rfloor$, and $\mu_{\lambda_{k}}=\mu \overline{\lambda_{k}}=1$, where $\lambda_{k}=m-i \cot ((\pi+2 k \pi) / 2 m)$, $k=0,1,2, \cdots,\lfloor m / 2\rfloor-1$.

By Theorem 2, $v_{0}=1, v_{m}=m-1$, and $v_{\lambda_{k}}=v_{\overline{\lambda_{k}}}=1$. We have the following:

$$
\begin{aligned}
& \text { if } m>1 \text { is odd, then } m=\mu_{m} \neq \nu_{m}=m-1 ; \\
& \text { if } m \text { is even, then } \mu_{\lambda}=v_{\lambda} \text {, where } \lambda=0, m, \lambda_{k}, \overline{\lambda_{k}} \text {, } \\
& k=0,1,2, \ldots,\lfloor m / 2\rfloor-1 .
\end{aligned}
$$

By Lemma 8(2), Corollary 4 holds.

\section{Conflict of Interests}

The author declares that there is no conflict of interests regarding the publication of this paper.

\section{Acknowledgment}

This work is supported by the Natural Science Foundation of Guangdong Province, China (no. S2013010016994).

\section{References}

[1] F. R. K. Chung, Spectral Graph Theory, vol. 92, AMS Publications, 1997.

[2] R. A. Brualdi, "Spectra of digraphs," Linear Algebra and Its Applications, vol. 432, no. 9, pp. 2181-2213, 2010.

[3] F. Chung, "Laplacians and the Cheeger inequality for directed graphs," Annals of Combinatorics, vol. 9, no. 1, pp. 1-19, 2005.

[4] W. N. Anderson, and T. D. Morley Jr., "Eigenvalues of the Laplacian of a graph," Linear and Multilinear Algebra, vol. 18, no. 2, pp. 141-145, 1985.

[5] R. Agaev and P. Chebotarev, "On the spectra of nonsymmetric Laplacian matrices," Linear Algebra and Its Applications, vol. 399, pp. 157-168, 2005.

[6] L. Bolian, Combinatorial Matrix Theory, Science Press, Beijing, China, 2nd edition, 2006.

[7] R. A. Brualdi and Q. Li, "Problem 31," Discrete Mathematics, vol. 43, pp. 1133-1135, 1983.

[8] S. W. Drury, "Solution of the conjecture of Brualdi and Li," Linear Algebra and Its Applications, vol. 436, no. 9, pp. 33923399, 2012.

[9] J. Burk and M. J. Tsatsomeros, "On the Brualdi-Li matrix and its Perron eigenspace," Electronic Journal of Linear Algebra, vol. 23, pp. 212-230, 2012.

[10] S. Kirkland, "A note on the sequence of Brualdi-Li matrices," Linear Algebra and Its Applications, vol. 248, pp. 233-240, 1996.

[11] S. Kirkland, "Hypertournament matrices, score vectors and eigenvalues," Linear and Multilinear Algebra, vol. 30, no. 4, pp. 261-274, 1991.

[12] S. J. Kirkland and B. L. Shader, "Tournament matrices with extremal spectral properties," Linear Algebra and Its Applications, vol. 196, pp. 1-17, 1994. 
[13] S. Kirkland, "Perron vector bounds for a tournament matrix with applications to a conjecture of Brualdi and Li," Linear Algebra and Its Applications, vol. 262, pp. 209-227, 1997.

[14] X. Chen, "On some properties for the sequence of Brualdi-Li matrices," Journal of Applied Mathematics, vol. 2013, Article ID 985654, 5 pages, 2013.

[15] Z. Herui, Advanced Algebra, Advanced Education Press, Beijing, China, 1983. 


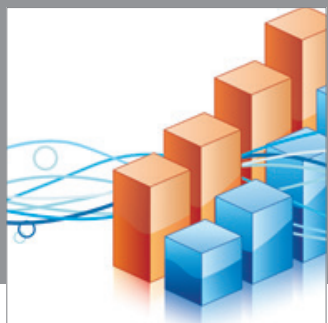

Advances in

Operations Research

mansans

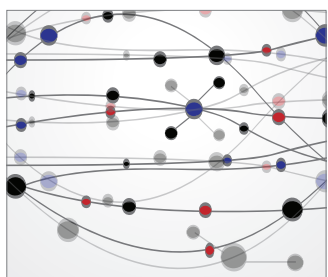

The Scientific World Journal
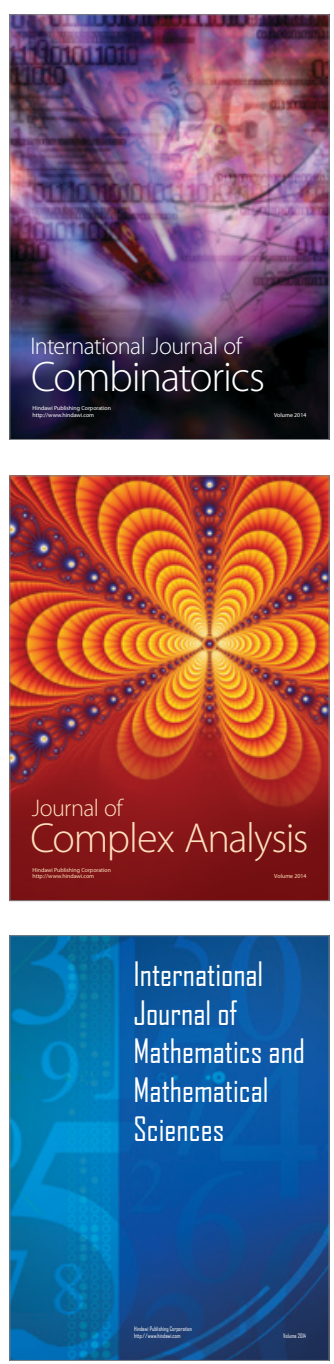
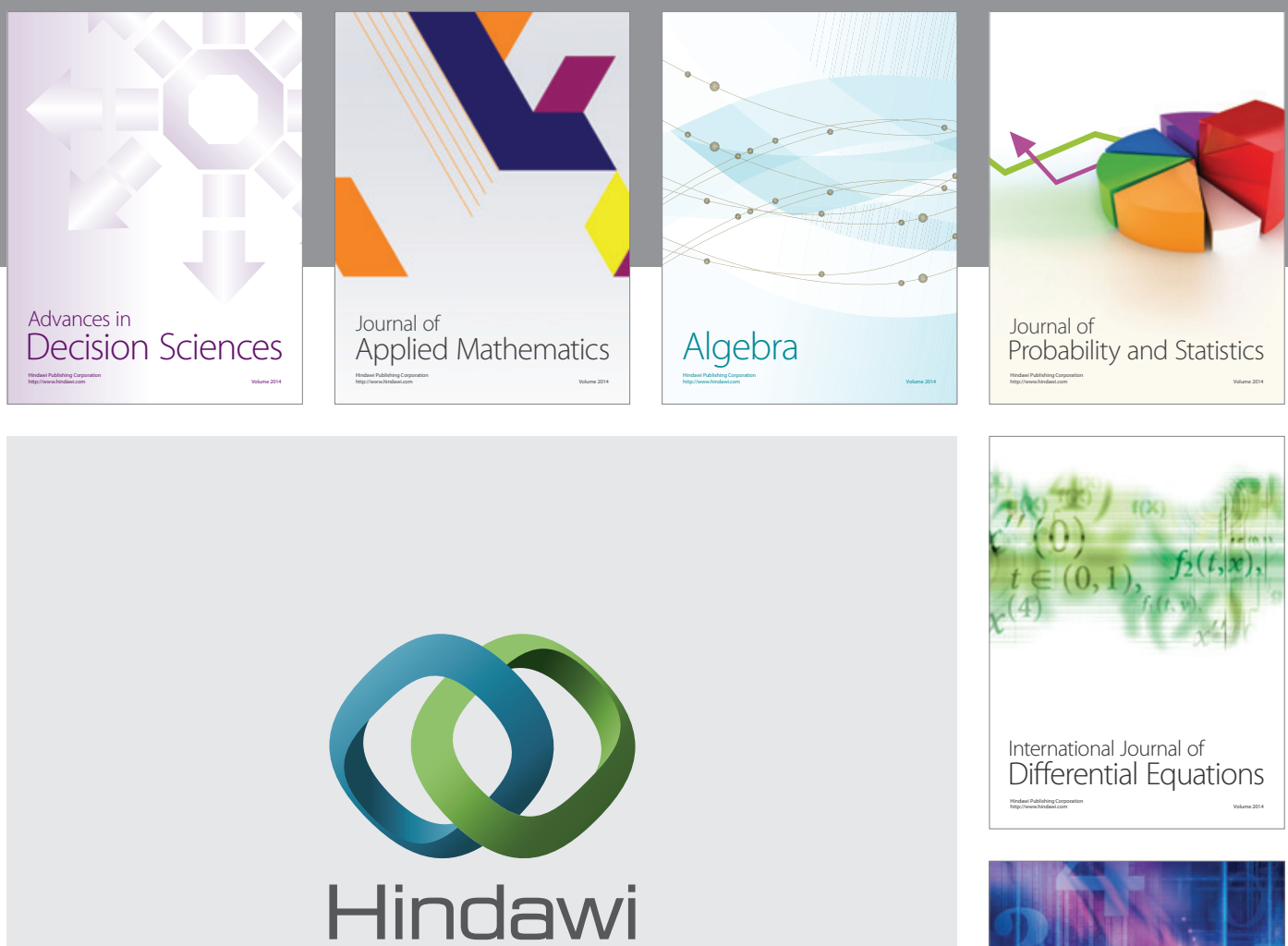

Submit your manuscripts at http://www.hindawi.com
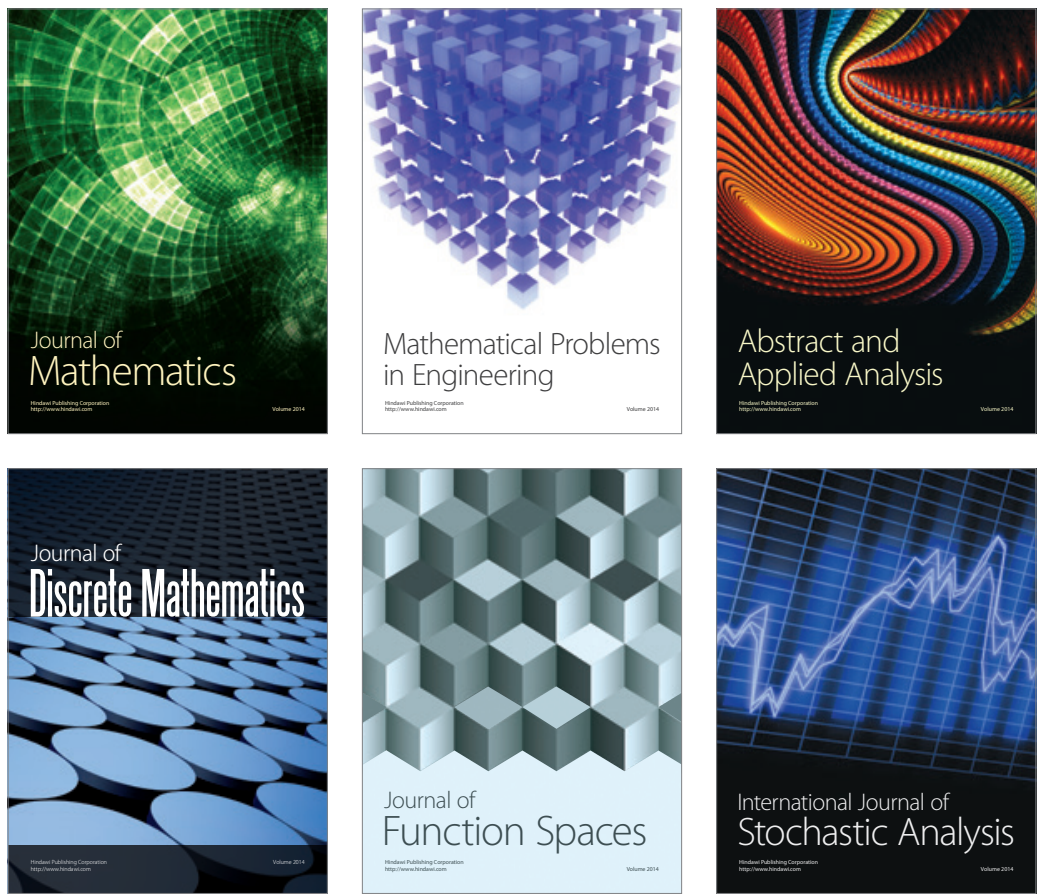

Journal of

Function Spaces

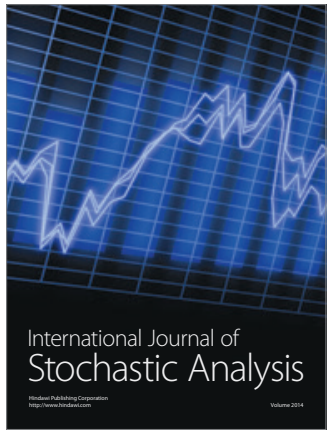


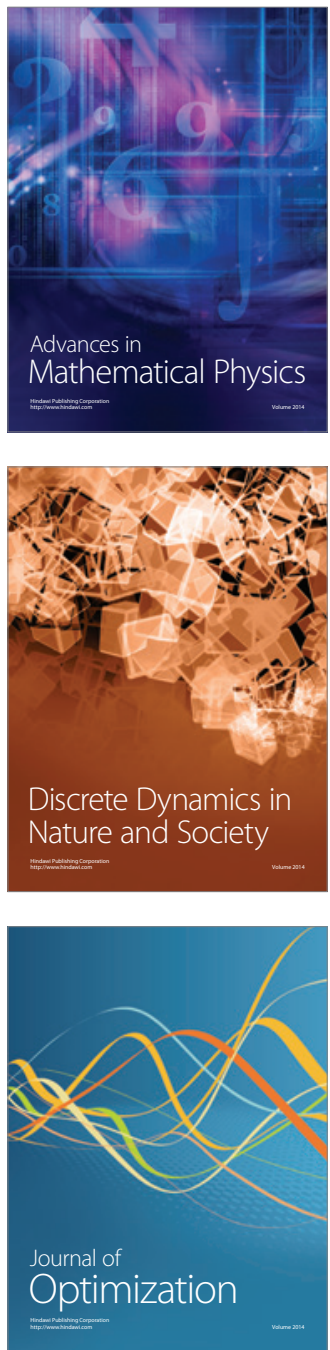